\title{
An Efficient Segmentation of Retinal Blood Vessels using Singular Value Decomposition and Morphological Operator
}

\author{
N. C.Santosh Kumar ${ }^{1}$, Y.Radhika ${ }^{2}$ \\ Department of Computer Science and Engineering, GIT, GITAM (Deemed to be University), Visakhapatnam, India
}

\begin{abstract}
The extensive study on retinal fundus images has become an essential part in medical domain to detect pathologies including diabetic retinopathy, cataract, glaucoma, macular degeneration,etc.which are the major causes of blindness. Automatic extraction of tree-shaped and unique retinal vascular structure from retinal fundus images is most exigent task and when achieved successfully, becomes a perfect tool helping ophthalmologists to follow appropriate diagnostic measures. In this work, a novel scheme to segment retinal tree-like vascular structure from retinal images is proposed using the Singular Value Decomposition's left singular vector matrix of the weighted $\mathbf{l}^{*} \mathrm{a}^{*} \mathbf{b}^{*}$ color model of the input image. The left singular vector matrix which captures the relevant and useful features helps in effective conversion of the input RGB image to gray image. Next, the converted gray image is contrast enhanced using CLAHE method which enhances the tree-shaped vasculature of the retinal blood vessel structure giving a rich contrast gray image. Further processing is carried out normalizing the contrast enhanced gray image by removing the image's background using a mean filter by which blood vessels become brighter. Later, the result of the difference between gray image and normalized filtered image is keyed-in as a constraint to perform ISODATA thresholding which globally segments the foreground vasculature from the image's background then followed by conversion of the resultant image into binary image upon which morphological opened operation is applied to take away small and falsely segmented portions producing accurate segmentation. This new technique got tested upon images contained in DRIVE and STARE databases and a performance metric called "area covered" is also calculated in addition with common metrics for sampled input image. This novel approach is empirically proven and has attaineda segmentation accuracy of $97.48 \%$.
\end{abstract}

Keywords-Singular value decomposition; left singular vector matrix; feature extraction; average filter; ISODAT Athresholding; morphological operators; stare anddrive databases

\section{INTRODUCTION}

Diabetes is such a disease that is affecting much and very rapidly deterioratinghuman's health, globally. Despite the advancements in the field of medicine, this disease will surely get amplified more and would ruin the happiness of the people irrespective of their age, sex, creed, etc. in the future. In automation of the discovery of blood vessels for a perfect analysis, the ideal blood vessels extraction is a complicated job as the appearance of the vessels takes indifferent diameter and width [1]. Thesequantifiable parameters of the varying vessel structure provide a clear specification of the kind of the ophthalmologic disease and its strength. According to [2], a huge number of patientshave lost their eye sight just because of the reach of diabetes on their retina. This spread of such disease is known formally as 'Diabetic Retinopathy'which isdetected by finding the discrepancies in the arrangement of retinal vasculature of retinal images. Most of the medical practitioners have suggested that early discovery and proper diagnosis of such irregularities that exists in blood vessels of retinal imagescouldlessenthe patients'suffering from vision failure. Blood vessels segmentation serves as a source for proper diagnosis of various ophthalmologic and cardiovasculardisorders that includes diabetic retinopathy, glaucoma, hypertension, etc. Vessel segmentation follows either supervised or unsupervised approach and is the primary footstep in performing computer aided diagnosis system development [3] that determines an ophthalmic disease. The outline, manifestation, and the direction of the tree-shaped retinal vasculature make the automation of segmentation a very challenging task. The issues relating to the contrast of the blood vessel structure and its background of the image, the noise presencein the image, and the anomalous composites upon retina like exudates, and lesions, microaneurysms, etc. makes the segmentation a tough task. In this effort, the proposed approach for segmentation ofblood vessels has workedupon varied sized retinal fundus color images and has been proven very efficient in performing the desired segmentation task. Thisunsupervised segmentation method has its roots from a mathematical technique which is well known as 'Singular Value Decomposition (SVD)'and has been exceptional in efficient extraction of blood vessels. These blood vessel features are captured in SVD's left singular vector matrix. Using these extracted features, the image preprocessing is carried out in which transforming the input RGB into grayscale image is done effectively. To overcome the major difficulties in working on green channel of the input retinal image, this successful attemptof using matrix algebra and vector calculus based Singular Value Decomposition is proven to be an outstanding one in image preprocessing phasewhich later makes life easier to moveontopost processing of the image using ISODATAthresholding and Morphological Operation producing an average segmentation accuracy of $97.48 \%$.

The remaining part of this paper is systematically organized with explanation of the proposed work in Section 2. The proposed method's empirical results are furnished in Section 3 with corresponding discussions. Section 4 gives the conclusion of the paper. 


\section{MATERIALS AND METHODS}

A non-invasive retinal fundus image capturing procedure involves penetration of red, green, and blue lights by the fundus camera into the human eye that eventually get absorbed back by the lens pigments differently. Among the three colors, the green channel in the RGB color image represents a rich contrast in image's blood possessing elements i.e. vessel structure than the background of the image. Most of the research which has been done had used the third portion of the input image i.e. green channel because it's a best and the finest way to exhibit vessels or background contrast. Also, it has the most light than other bands to which human eye is very much sensitive as compared to the red channel, which does possess the brightest color and have low contrast. Other side, blue channel suffers and bear deprived dynamic range [4].But, sometimes green channel also creates hidden noise in the image due to variations in the intensities that infers blurriness in the image and in turn become a challenging task to overcome.The other important reason is that the blood vessels possess very meager local contrast and to overcome this problem in processing an image, various imaging filters have been applied so far.To have an alternative and a replacement to retinal image's green channel, a new procedure has been devised for proper transformationto gray imagefromRGB color image with the help of matrix algebra and vector calculus related mathematical technique called "Singular Value Decomposition (SVD)" which is computed upon input image and later weighted with well-known $1 * a * b$ color model. In this paper, left singular vector matrix which is one among the decomposed matrices of the computed Singular Value Decomposition (SVD) (where SVD takes in $l * a * b *$ processed input image as input) using the built-in function in MATLAB, captures the required features of blood vessels and arranges them in the decreasing order as the column vectors based on the light intensities provided by the $1^{*} \mathrm{a}^{*} \mathrm{~b} *$ while keeping the non-blood vessels as zeroes simultaneously. This separation and arrangement of blood as well non-blood vessels is one of the magnificent task performed by decomposed left singular vector matrix of SVD.

The sequence of steps depicted in the flowchart is shown in Fig. 1. It gives the step-by-step tasks performed in blood vessels extraction. After reading the input image, it is modeled to $1 * a * b *$ color model upon which Singular Value Decomposition (SVD) is computed to capture the required feature set of blood vessels as the ordered column vectors in the decomposed left singular vector matrix. Such feature set which is held by the left singular vector matrix is then contrast enhanced using CLAHE. Later, a mean filter is been used to exclude image's background from the contrast enhanced image. Then, the difference between the contrast enhanced image and filtered image is computed to be used as a threshold level parameter for the ISODATAthresholding which automatically finds a good threshold level to help binarize the whole gray image for segmenting the foreground tree-shaped blood vessel structure. Finally, an open morphological operation removes the smaller sized and unwanted vessels producing a perfect and required segmentation result.

\section{Proposed method's algorithm steps:}

The following are sequence of steps of the proposed procedure for segmentation of blood vessels:

Step 1 Read input image

Step 2 Using Singular Value Decomposition convert RGB to gray image

Step 3 Apply CLAHE for contrast enhancement of the gray image ofStep (2)

Step 4 Exclude background of the image by applying Averaging Filter with 9x9 mask.

Step 5 Compute the difference image of Step (3) and Step (4) images to be used as a parameter for ISODATA thresholding which is computed in the Step (6).

Step 6 Find the threshold for the image obtained in Step (5) using ISODATA thresholding Method

Step 7 Convert the resultant image of Step (5) to binary image using the computed threshold of Step (6).

Step 8 Remove small pixels using morphological open operator on the resultant image of Step (7).

Step 9 Realize the segmented image

\section{A. Singular Value Decomposition (SVD)}

Any real and a square or a rectangular matrix $D$ in linear algebra, could be decomposed perfectly as a product of three distinct matrices $\mathrm{D}={ }_{P Q R}{ }^{T}$, where matrices $P$ and $R$ are termed as orthogonal matrices i.e. $P^{T} P=\mathrm{I},{ }_{R}^{T}{ }_{R}=\mathrm{I}$ and the singular matrix is $\mathrm{Q}=\operatorname{diagonal}(\mathrm{q} 1, \mathrm{q} 2 \ldots)$. Here, the diagonal entries are D's singular values and each column in P is the D's left singular vectors, and each column in $\mathrm{R}$ is the D's right singular vectors. This helpful breakdown of a single matrix into three is called as Singular Value Decomposition (SVD) [5]. Generally, this SVD can be used in three ways in any real world application.

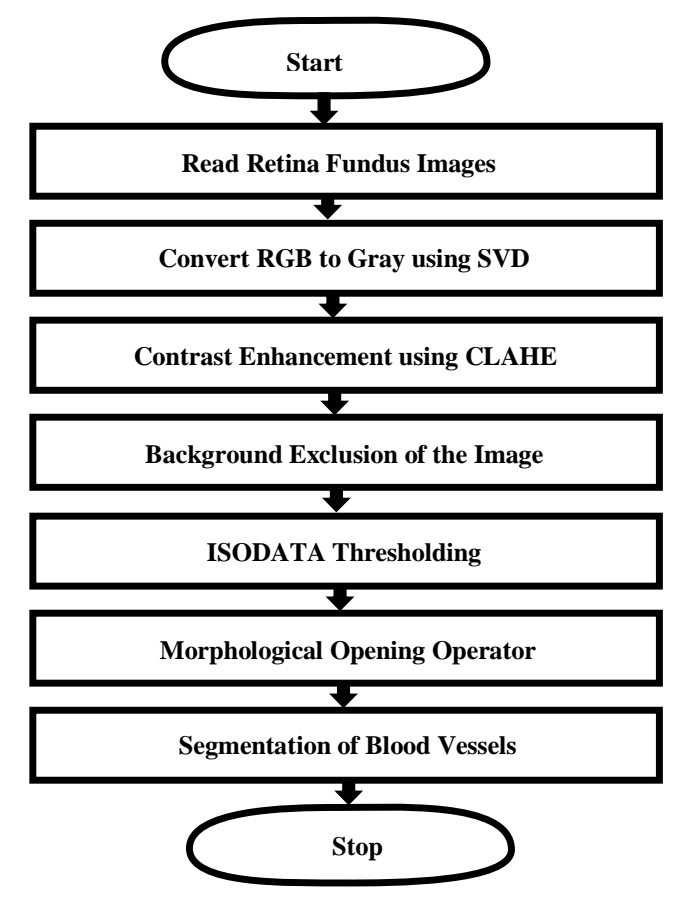

Fig. 1. Proposed Method's Flowchart. 
a) Firstly, it can be utilized as a processthat transforms interrelated variables or objectsinto a set of unrelated objects or variables that are used to expose a variety of relationships that exists within data under consideration.

b) Secondly, SVD can be used as a perfect means for recognizing and sorting the dimensions of those data elements which show the most deviation.

c) The third form of using SVD is to best approximate the capturedand most deviated data points by means of smaller number dimensions. Thus, SVD could be a best scheme for data reduction.

From the above three primary uses of SVD, this paper uses the second benefit in form of left singular vector matrix to identify and arrange the dimensions(column vectors of the image) that reveal the deviations of blood and non-blood vessels. For reader's perception, a detailed discussion on the background of matrix algebra and vector calculus is followed next.

\section{B. Matrix Algebra and Vector Calculus of SVD}

Matrix algebra and vector calculus are two very important mathematical areas for data analysis as well as for statistical theory. Here, this part of the paper deals with introducing the background of vectors and matrices that are applied perfectly to the digital image processing because an image is a $2 \mathrm{D}$ function which is a matrix or a two-dimensional array representation in computer memory. Vectors are a series of numbers along various dimensions where those numbers which comprising the vectors are known as components and these can be the measurements of a real-world object in consideration. The components' count represents the dimensionality of that vector. The intuitive in mathematical notation is that the vector $V$ with m-dimensions is a series of m-numbers as such the component $v_{i}$ is the value of the vector $V$ on $i^{\text {th }}$-dimension.

Suppose matrix $A$ is a table of rows and columns which does have two subscripts i.e. $n, m$ which are the maximum count of rows and columns respectively for that matrix. The matrix's entries are called 'components' or 'elements' which are represented by a lower-case letter ' $a$ ' and the specific entry of the matrix can be located by means of row index which is labeled as $i$ and the column index which is labeled as $j$. Moreover, an identity matrix can be defined as a square matrix having elements on the diagonal as 1 with remaining other entries as 0.Let the RGB input image under consideration be matrix A possessing various image intensities as column vectors. This image matrix $A$ is transformed into $1^{*} a^{*} b^{*}$ color representation upon which SVD is then computed resulting in three distinct matrices, namely:

a) Left singular vector matrix

b) Diagonal matrix

c) Right singular vector matrix

Mathematically, this is shown in equationbelow

$A_{m n}=U_{m n} S_{m n} V_{n n}^{T}$

where, A is the matrix with ' $m$ ' rows and ' $n$ ' columns' which can be decomposed as the product of three matrices namely (i) matrix U is Left Singular Vector matrix with same order of matrix A (ii) Matrix S is the Diagonal matrix with same order of A (iii) Right Singular Vector matrix is with ' $n$ ' rows and ' $n$ ' columns.

As this paper made use of the left singular vector matrix, the only focus is kept on the discussion of left singular vector matrix.

\section{Left Singular Vector Matrix}

A matrix $U$ is an orthogonal matrix that is computed on a given input image matrix $A$ and possesses column vectors which areeigenvectorsof $A A^{T}$ and these vectors are orthonormal.

The detailed explanation of the terms involved in the definition of left singular vector matrix is followed:

\section{Orthogonal Matrix}

Any matrix is said to be orthogonal matrix if it satisfies the following criteria:

a) Orthogonal matrix should be a square matrix with same count of rows as well as columns. Hence the left singular matrix has rows and columns

b) Matrix $U$ is orthogonal if

$U U^{T}=U^{T} U=I$

Identity matrix is termed to be a square matrix with component entries on its diagonal are all equal to 1 remaining all other component entries as zero.A matrix $A$ is orthogonal matrixif.

$$
A^{T}=A^{T} A=I
$$

Similarly, a diagonal matrix $A$ contains nonzero values in the main diagonal from its upper left towardsits lower right corner compulsorily and includes all other elements as zeroes. The square matrix's determinant is such a function that condenses that matrix to a particular single number.

\section{E. Orthonormal}

Vectors having a length of 1 and are perpendicular with each other are orthonormal. This single word is a combination of two distinct words orthogonal and normal. Any two vectors either insquare or in a rectangular matrix are supposedto be orthogonal (or perpendicular) if their inner product is zero. Such vectors are perpendicular to each other which mean the angle between them is $90^{\theta}$. Simultaneously, a unit or normal vector is the one whose length is 1 . Any vector with a length greater than zero can be made normalized by simply dividing every element by its length. Thecalculation of vector's length is done by squaring each element of the vector, addingall of them, and then computing the square root value of the sum.For example, if we consider $\vec{v}$ as a vector then its length denoted by $|\vec{v}|$ is computed using the equation given below:

$|\vec{v}|=\sqrt{\sum_{i=1}^{n} X_{i}^{2}}$ 
The addition of vectors can be computed by adding corresponding components or elements of similar positions in both vectors resulting in a new vector. In general representation, the vector's addition is given by the equation (3) which is stated below:

$$
\begin{aligned}
& \text { If } A=\left[a_{1}, a_{2}, \ldots \ldots . ., a_{n}\right] \text { and } B=\left[b_{1}, b_{2}, \ldots \ldots ., b_{n}\right]_{\text {then }} \\
& A+B=\left[a_{1}+b_{1} a_{2}+b_{2}, \ldots \ldots ., a_{n}+b_{n,}\right]
\end{aligned}
$$

A scalar value is any real number can be multiplied with a vector implies multiplying that scalar value to every component or element of that vector which produces a new vector. Scalar value multiplication means if $d$ can be any realnumber and $\vec{v}$ can be any vector containing elements as $\left[x_{1}, x_{2}, \ldots \ldots ., x_{n}\right]$ then a new vector is yielded as shown below in equation (4)

$$
d * \vec{v}=\left[d x_{1} d x_{2}, \ldots \ldots . ., d x_{n}\right]
$$

Two vectors having same dimensions can be multiplied with each other and the result is known as inner product. This can bedone by multiplying corresponding component of one vector by the same positioned component of other vector and adding all of them together is yielding a scalar value. When this scalar value is zero, then the two vectors involved in multiplication are perpendicular or orthogonal to each other. This inner product is also called as scalar or dot product. The two vector's inner product is denoted as

$$
\left(\vec{v}_{1}, \vec{v}_{2}\right)_{\text {or }} \vec{v}_{1}, \vec{v}_{2} \text { and generally denoted as below equation: }
$$$$
(\vec{X}, \vec{Y})=\vec{X} \cdot \vec{Y}=\sum_{i=1}^{n} X_{i} Y_{i}
$$

\section{Eigenvectors}

An eigenvector is a vector that is non-zero and fitsin the following equation (6):

$A \vec{v}=\lambda \vec{v}$

where $A$ represents a square matrix, $\lambda$ denotes a scalar value or real number, $\vec{v}$ is the eigenvector. By taking matrix as an arrangement of linear equations, the eigenvalues and eigenvector can be found by cracking for those variable values that solely gives the elements of the eigenvector.

Hence, from above discussions upon the theoretical concepts of matrix algebra and vector calculus, it is very elegant and evident that these mathematical techniques play a vital role in image processing, especially, in the preprocessing phase for extraction of the required feature set from given input image.

\section{F. The RGB Color Representation}

For proper representation upon computer display, various proportions of red color, green color and blue color are combined to obtain different colors that are in the horizons of visible spectrum. The percentage range of levels of intensities of these three dominant colors is 0 to 100 . The color modelRGBcan be manifested with the following intensity function:

$$
I_{R G B}=\left(F_{R}, F_{G}, F_{B}\right)
$$

where $F_{R}(x, y)$ corresponds to the pixel's intensity in the red component, $F_{G}(x, y)$ and $F_{B}(x, y)$ symbolizes the pixel's intensity in green color as well blue components respectively. The words in [6]mentioned that the color component values get stored in the computer as integer numbers that span 0 to 255 ranges; this is the range that is offered by a single byte which is regularly represented as decimal or hexadecimal numbers. RGB values get encoded as 8-bit integer numbers, which is of 0 to 255 ranges. The intuition here is that the three colors takes 24-bit format meaning that of 24-bit format distributed as 8 bits each for red, for green,for blue. Based on the choice, an adjustment can be made in the quantity of the three portions to derive any of the other colors. For example, if one considers the decimal code for RGB colors, it takes triplet form such that red color in that triplet takes a value of 255 and other two colors as zeroes. This type of decimal coding is applied for green as well for blue color. In order to generate a black color, the decimal code triplet values are all zeroes, for white color the triplet values are all 255 and for gray it is set as $(128,128,128)$. In this paper, images with high-resolution are taken as input for blood vessels segmentation.

\section{G. The Gray Image}

A gray scale image is a matrix with integer numbers as pixel valuesbased on the light intensities of an image that correspond to either black or white color. A grayscale image in MATLABgets stored as an individual matrix where every element in such matrix corresponds to the pixel of an image. The author in [7] discussed that the image's constructionis done using sophisticated sensors and with other image acquirement equipment that signify the brightness (otherwise called as intensity) $I$ of image's light as 2Dfunction which is continuous and is represented as $F(x, y)$ for which $(x, y)$ stand forspatial attributes and whose value corresponds the light's brightness. The matrix can span from class uint8 to double where class uint16, class int16, class single stand between them. The values between $[0,255]$ for uint8, for uint16 [0, 65535], and [-32768, 32767] for int16 are the respective class ranges. For a single or double matrix class, while using the grayscalecolor map, black is represented by 0 intensityand white is represented with intensity 1 . For matricesspanning type uint8 to int16,black is represented with intensity intmin(class(I)) and representation of whitewith intensity intmax(class (I)). In this paper, a mask is tailoredbased on Singular Value Decomposition which is used in getting a grayimage from RGB as a preprocessing step.

\section{H. $l * a * b *$ Color Model}

According to [8], as for identifying a site location having geographic coordinates such as longitude, latitude, and altitude is very similar to have a way to locate and communicate colors using $1 * a * b *$ color values where the letter 1 represents 
lightness, the letter a represents red/green, and the letter b indicates blue/yellow. During 1940's, Richard Hunter who commenced a tri-stimulus model famously called as $L a b$ have given a scaling to attain close unvarying gap of apparentdifferences in color. The Labwas made as a benchmark for perfectly plotting complete coordinates of colors and their differences. The $1^{*} \mathrm{a}^{*} \mathrm{~b} *$ or Lab color model indicates the color values for the input image. In this work, color space $1 * a * b *$ is used as an intermediary model to make a perfect inline in the process of conversion to gray image from input RGB image.

\section{Converting into Gray Image from RGB Image}

Gray scaled images havecontinuous range of gray values while a binary image has only two possible values for each pixel. Grayscales are represented as integers within the computer. A clear presentation in [9] has given factsabout the pixel value's luminance of animage whose values ranges between 0 to 255 . Here, the value 0 denotes black, 255 for white and the values in between 0 and 255 takes the various shades of gray.The process of conversioninto a gray image from RGB image is typically changing the values of RGB (which are of 24-bit) into values of grayscale (which are of 8bit). The RGB image contains three components and can be taken into consideration as three different images having three scales as red, green, blue that are stacked on top of each other. The array of the orderM*N*3 of color pixel is for RGB image, whereas agrayscaled image is a single layered image that takes shape as $M^{*} N$ array whose pixel values represents intensities. A readily available off-the-shelf function in MATLAB called 'rgb2gray()'iscommonly used to changea RGB image to grayimage. In order to save a single-color pixel of an RGB, we would require $8 * 3=24$ bits ( 8 bit for each color component), but during the conversion to grayscale image from RGB image, only 8-bits are required to preserve an image's single pixel.

\section{RELATED WORK}

In the literature, a handful of algorithms were proposed to convert into 8-bit gray image from 24-bit RGB image. According to the paper [10], the authors have pioneered to preserve the contrast, sharpness, shadow, and the structure of the image which performs approximation, addition, reduction of luminance and chrominance. The algorithm which was implemented in MATLAB had extracted the three color components into three 2-D matrices and had accumulated the weighted pixel values at corresponding positions of 2-D matrices into a new matrix of the image size. This new matrix holding the weighted sum of each pixel of three color components formed the gray image. The author Tyler Coye in [11] had proposed a new technique which he developed in MATLAB to convert RGB image to gray where he used Principal Component Analysis (PCA) technique to compute principal components or vectors of the input image and then created a weighted mask that got applied on PCA transformed image for convertinginto gray image from RGB image.In the paper [12], the authors proposed a conversion technique depending upon the principle to preserve color image's visual perception. The method proceeds focusing on the (i) chromaticity contrastdistinctiveness of pixels in the color image rather thanthe luminance contrast, (ii) Region-based contrast which instead of individual pixels works upon the regions of the image (iii) Distance-dependent contrast convey the meaning that there should be inverse proportion of the distance between two regions (iv) Saliency Preservation where saliency(features) of the color image needed to properly preserved in the resulting grayscale image. This method had achieved the required aforementioned objectives by formulating anaugmented mapping function for color-to-gray that targets to map each pixel's color of the input image $I$ to the grayscaled value to form the resulting gray image which is given below:

$$
\operatorname{gray}_{p}=L_{p}+C_{p}
$$

where gray $_{p}$ represents the resulting gray color value of the pixel $P$ in $I, L_{p}$ is $P^{\prime} s$ value of luminantwhich is independent of the locationis calculated using the below mentioned equation:

$$
L_{p}=L_{p}^{*}+\left(0.1340 q(\theta)+0.0872 K_{B r}\right) S_{u v} L_{p}^{*}
$$

where $q(\theta)$ is the quadrant metric, $S_{u v}$ is the chromatic saturation, and $K_{B r}$ is a constant.

$C_{p}$ is the $P^{\prime} s$ value of the luminant which is not only location-dependent factorbutalso plays a vital role in adjusting $L_{p}$ accordingly upon the color variations of $P$ which is calculated using the below mentioned equation:

$C_{p}=k \sum_{i=1}^{n}\left(a_{p}-a_{q}\right) e^{\frac{-\Delta D_{p q}}{\sigma^{2}}}+l \sum_{i=1}^{n}\left(b_{p}-b_{q}\right) e^{\frac{-\Delta D_{p q}}{\sigma^{2}}}$

where, $a_{p}$ and $a_{q}$ are the A channel(the authors of the paper got motivated by chromaticity-based contrast and have measured the differences in chrominance by a combination of two distance-weighted color differences of two channels where they named them as A channel and B channel of CIELAB color space) values of pixels $\mathrm{p}$ and $\mathrm{q}$ respectively. Similarly, $b_{p}$ and $b_{q}$ are the values of $\mathrm{B}$ channel corresponding to and $q . D_{p q}$ is the Euclidean distance between $p$ and $q$. The exponent $\mathrm{e}$ is used to approximate the location-dependent contrast. Also, $\sigma^{2}$ is been set with an fuzzy value between 0 and 1 depending on the width and height of the image. Here, $k$ and $l$ are variables which are unknown and have been optimized depending on the criteria of saliency preservation. $n$ is the size of the pixels in the image.

In this paper, the approach followed is very specific where a mask is created upon the calculated left singular value matrix of the $1 * a * b *$ transformed input RGB image. The mask used here operates upon every organized pixel of Left Singular Vector matrix to convert them to take values of gray. This new 
technique has been developed using MATLAB software. The steps involved in preprocessing the image which is performed after the input image is read, resized for easy computation, and get doubled which holds real values between 0 to 255 of the image, are mentioned below:

Step 1 Translate the doubled image to $1^{*} \mathrm{a} * \mathrm{~b} *$ color model.

Step 2 Compute the new image with the weighted combination of the vectors of the resultant $1 * a * b *$ model image of Step (1) in order to increase the contrast in the gray version of those partswith different hues but having similar intensities.

Step 3 Decompose the image of Step (2) using SVD into three matrices namely: Left Singular Vector Matrix, Diagonal Matrix, and Right Singular Vector Matrix.

Step 4 Extract Left Singular Vector Matrix of the SVD which holds the feature set in column vectors of decreasing order.

Step 5 To have the size compatibility, reshape Left Singular Vector Matrix to fit to the size of resultant image of Step (1) such that the required feature set can be accessed as the first portion with that size.

Step 6 Access the featured column vectors and normalize it with maskthat works on the every pixel of the column vector. The mask operates on each pixel where at each pixel the computation takes place as the ratio of the computed result of subtraction ofpixel of the matrix with the least pixel with thecomputed result of subtraction of maximum pixel with least pixel of the matrix. The outcome of this step is the gray version of the given input RGB image.

The gray scaled image, the outcome of the conversion procedure disclosed above, is fed as the input for contrast enhancement to the CLAHE which is discussed next.

\section{A. AbbreviationsAdaptive Histogram Equalization (AHE)}

A perfect analysis of an image needs a perfect preprocessing of it using contrast enhancement technique which can be done locally or globally. The study done by [13] revealed that the usage of CLAHE which is based on local contrast enhancement had given optimal results which when compared to other global enhancement schemes namely histogram specification, BSB-CLAHE, histogram equalization, AHE that mostly get applied on colored image segmentation. The authors in paper [14] have utilized the same CLAHE along with other Wiener filter method to enhance the image's contrast. Usually, they used filters to take out the noise there in image and to shift image into appropriate dynamic range they used Gamma correction techniques. Finally, they went with CLAHE that avoided amplification of unnecessary noise hidden in the image and have devised its parameters to constrain the contrast in identical areas. Image sharpness can be increased by magnifying image's contrast which is one of the important tasks in a system of automated disease diagnosis.Contrast Limited Adaptive Histogram Equalization (CLAHE) is animage processingtechnique which is an extended edition of adaptive histogram equalization (AHE). The computerized procedure AHE is familiar in the realm of image processing which is primarily used to amplify the image's contrast. This technique which works on small subdivisions rather than whole image, initially, computes histogram for every region and then redistributes the lightness values to that particular region. Despite making improvements in the image's local contrast, this method has huge chances to make strong the noise in those regions that are relatively homogeneous of that image. To overcome this, CLAHE is used to act globally and limiting the contrast. In this paper, preprocessing of the image is done using CLAHE which takes SVD transformed gray image as a parameter and ideally enhances the contrast of those relevant features which are being captured by left singular SVD matrix.

\section{B. Averaging Filter for Smoothing}

To reduce the intensity variation among pixels and to reduce noise that is present in the input image, filters are extensively used and are the facilitators in the domain of processing of the image.Mean filter or an average filter is windowed and linear class filters that is easy to implementto smooth the given image. Such filters usually works as best low-pass one. For any element of animage, take an average across its neighborhood based on the size of the mask chosen, for say, $3 \times 3$ or $5 \times 5$ or $9 \times 9$, and replace the average value to that location of the element is a very simple idea behind this mean filter.

\section{ISODATAThresholding for Segmentation}

The simplest segmentation method in image processing is thresholding. This method splits the given image into minor segments using a single color scale named gray scale value for properly defining boundary of each. The advantage behind getting a binary image is realized in decreasing the given input data'sdifficultyand extensively simplifying the recognition of the object and its classification processes [15]. The pixels get partitioned based on their intensity value.Once having thegrayscale image as a parameter to this method, then thresholding easily creates images that are binary. The process of this technique moves by considering each individual pixelthat isscored based on the value compared to the desired threshold value. The pixel is an "object" pixel if the score is greater than the given threshold and is "background" pixel if the score value falls below the threshold value. During this process of deciding on whether a pixel is object or background, the replacement is made with 1 if it is an object pixel and with 0 if it is a background pixel. ISODATA classification scheme computes the class 'means'of the data space that is evenly distributed in it. Then, it proceeds with an iterative approach to cluster all other remaining pixels with the help of techniques that calculates minimum distance. This algorithm recalculates 'means'at every iteration and reclassifies all other remaining pixels accordingly aligned with the new means being computed. Based on the input threshold parameters, this technique performs iterative splitting of the class, merging, and deletions if required. Here, classification of every pixel to its nearest class is done unless specification is made for a standard deviation measure or for the value of the distance threshold. If went unspecified, some pixels may have the chances to get forego classification once the selected criteria is not met by 
them. This procedure iterates until pixels countin every classgets changedwhich should be at most the value of the chosen pixel change threshold or until the maximumiterations specified is reached [16].

In this paper, ISODATA(Iterative Self-Organizing Data Analysis Technique) thresholding is performed by taking resultant subtracted image that is being computed between gray image and mean filter image as an input parameter.

\section{Morphological Area Open Operation}

According to [16], theoperations that span a broad set in the domain of image processing as well as works upon the object'sshapes in the given input image is carried out by Morphological Operators. These operations of the operators which apply a structuring element to createa desired and same sizedoutput image are very much familiar to the researchers who witness their effort in segmentation of images. The operation of these operators depends on getting the corresponding pixel value in the output image where each output pixel results from the input pixel value after being subject to work on with its neighboring pixel values based on the given criteria. Mostly on binary images these morphological operators are used, e.g., for background subtraction from an image. They are used on gray value images, if they are viewed as a stack to binary images. Most of the operations used in image processing are a combination of two processes, dilation and erosion. Moreover, the two forms of structuring element which work on binary images and gray images respectively are flat and non-flat. In the paper [17], morphological area open is used toremove all of the connected components that do have fewer pixels than the given parametric pixel size from the binary image which is the offset parameter to this method which outputs in a binary image as the final segmented image.The morphological opening is very useful for conserving the object's shape and size along while removing small unwanted objects. The mathematical formula for the morphological opening is given below in equation (11) which is the calculation of dilation from the calculated erosion where erosion works upon a set of ' $A$ ' by using a structuring element ' $\mathrm{B}$ 'which is defined as

$$
A o B=(A \square B) \oplus B
$$

where $\square$ denotes erosion and $\oplus$ denotes dilation.

In this proposed work, the morphological opening operator has been used to remove small-sized and unwanted blood vessels while safeguarding the blood vessels shape and size. Here, the function takes two parameters: (i) Flat structuring element parameter: the value of this parameter is the binary image which is computed after the ISO thresholding method discussed above (ii) Offset length: the value of this parameter is an integer number which is set between 20 and 100 for the images being worked on. This offset length is crucial as the removal of the blood vessels depends upon the value been set.

\section{RESUlTS AND Discussions}

\section{A. STARE and DRIVEDatabases}

1) STARE database:There is another database containing retina color fundus images by name STructured Analysis of the REtina (STARE)[19].The training dataset relating to blood vessels segmentation contains 40 manually segmented images performed by two observers and are subdivided into two sets where the first set is termed as first observer and the second one as second observer. Test dataset have 20 color images: 10 usual and 10unusual which are beingtaken by a TopCon TRV50 fundus camera atFOV of $35^{\circ}$ and the image's size is $700 \mathrm{X}$ 605 pixels.

2) DRIVE database:DigitalRetinal Images for Vessel Extraction (DRIVE) database [18] is such a database introduced in the year 2004 to serve the perfect purpose in carrying out an ideal research work in the field of retinal fundus image processing. There are about 40 retina images among them 20are training images and other 20 are test images which are being captured by nonmydriatic3CCDCanon CR5 camera at 45_ field of view (FOV). Theimage's size is of 768 x 584 pixels where it takes 8-bits to each colorchannel. Also, this database contains both normalas well as abnormal images along with manuallylabeled images by experts to make an assessment of the proposed method's performance.The most important aspect of this database is the inclusion of the masks for separation of FOV from image's remaining part.Another aspect of this database is regarding theavailability of two hand labeled setsin which thefirst set provides the ground truth for results evaluation of any proposed manual while the second set containshalf of the images which are hand labeled.

\section{B. Performance Measures}

The method which is proposed in this paper has been implemented inMATLAB 2017Bb andthe assessment of the performanceisdone in view of its specificity, sensitivity, and accuracy. The proposed method is implemented on DRIVE and STARE database images. This method has utilized the Singular Value Decomposition (SVD) for effective conversion of RGB to gray, followed by adaptive histogram equalization (AHE) for good enhancement results. After having a good contrast enhancement, the effective segmentationis followed by ISODATAthresholding. Here, the thresholded segmentation results havesuffered from some boundary leakages and unwanted pixels presence. In order to remove these drawbacks of thresholding method, the morphological opening is used to prune away the small and needless blood vessels finally producing a best segmented image as output.The metrics used in analyzing the performance of the proposed technique are:

$\begin{aligned} \text { Sensitivity }= & \frac{T P}{T P+F N} \\ \text { Specificity }= & \frac{T N}{T N+F P}\end{aligned}$

Accuracy $=\frac{T P+T N}{T P+T N+F P+F N}$

True Positive (TP) give the count of pixelsin the image being segmented and also in the groundtruth.False Positive 
(FP) gives the count of pixels detected in the image under consideration but not inground truth of the same image.Trueratio is defined as the ratio of the count of true pixels belongs to blood vesselsand the countof ground truth's vessel pixels. Finally, falseratio is the ratio of non-blood vessel pixels count to thecount ofground truth's non-vessel pixels.

\section{Performance Comparison of Existing and Proposed Methods}

The segmentation performance of the proposed method is analyzed based on the parameters such as sensitivity, specificity and accuracy. The performance measures are calculated based on the equations (12,13 and 14). The total count of the pixels properly classified as the vessel (true positives) pixel and non-vessel pixel(true negatives) divided by the total count of pixels in the images determines the accuracy of the segmentation algorithm. Moreover, the measurement of the segmentation correctness is obtained through true positive rate (TPR) and false positive rate (FPR). TPR is the result of the ratio of total count of true positives to the count of true blood vessel pixels. The FPR is the outcome of the ratio of the total count of false positives to the count of non-vessel pixels spotted in the ground truth image.

The performance measures of the existing blood vessels in retinal images as shown in the Table I. From the Table I, first eight rows show the performance of existing segmentation for blood vessels. The maximum segmentation accuracy of existing methods is such as Youetal (2011),Kumar et al (2016), Soares et al (2006),Mendonca et al (2006) , B. Zhang et al (2010), Martinez-Perez et al (2007), Mendonca and Campilho et al (2006), Palomera-Perez et al. (2010)were achieved an accuracy of $94.65 \%, 96.26 \%, 94.80 \%, 94.66 \%, 93.82 \%, 93.44 \%$ , $94.52 \%$ and $92.55 \%$ respectively.

In the literature survey on blood vessels segmentation algorithms, it is found that the pre-processing stage plays a crucial role to get the effective segmentation accuracy. So, the proposed algorithm does the pre-processing of images by using singular valued decomposition (SVD) for RGB to gray scale effectively. This algorithm is tested on four different images that are taken from databases mentioned. After successful testing and assessment, the outstanding performance is witnessed in the parameters such as sensitivity, specificity and accuracy of the proposed method tabulated in last row of Table I. Moreover, segmentation accuracy of the proposed method is $97.98 \%, 98.45 \%, 98.32 \%$ and $98.78 \%$ of four images as shown in Fig. 2, 3, 4 and 5, respectively. Finally, based on the performance analysis done in the Table I, the proposed method segments the retinal blood vessels accurately and effectively of $97.48 \%$ compared with the existing blood vessels segmentation algorithms.

Fig. 2, 3, 4 and 5 also show the results of proposed method, Fig. 2(a), 3(a), 4(a) and 5(a) are the input retinal blood vessels fundus images, Fig. 2(b), 3(b), 4(b) and 5(b) are the effective RGB to Gray conversion images by using SVD, Fig. 2(c), 3(c), 4(c), and 5(c) are the subtracted images of gray and average filter, Fig. 2(d,e), 3(d,e), 4(d,e) and 5(d,e) are thefinal blood vessels segmentation and its perimeter images respectively. The result analysis of the proposed approachis provided in Table I. Table II gives the segmentation area of the blood vessels covered by the proposed method.

\section{Experimental Results on Various Blood Vessels in Fundus \\ Images}

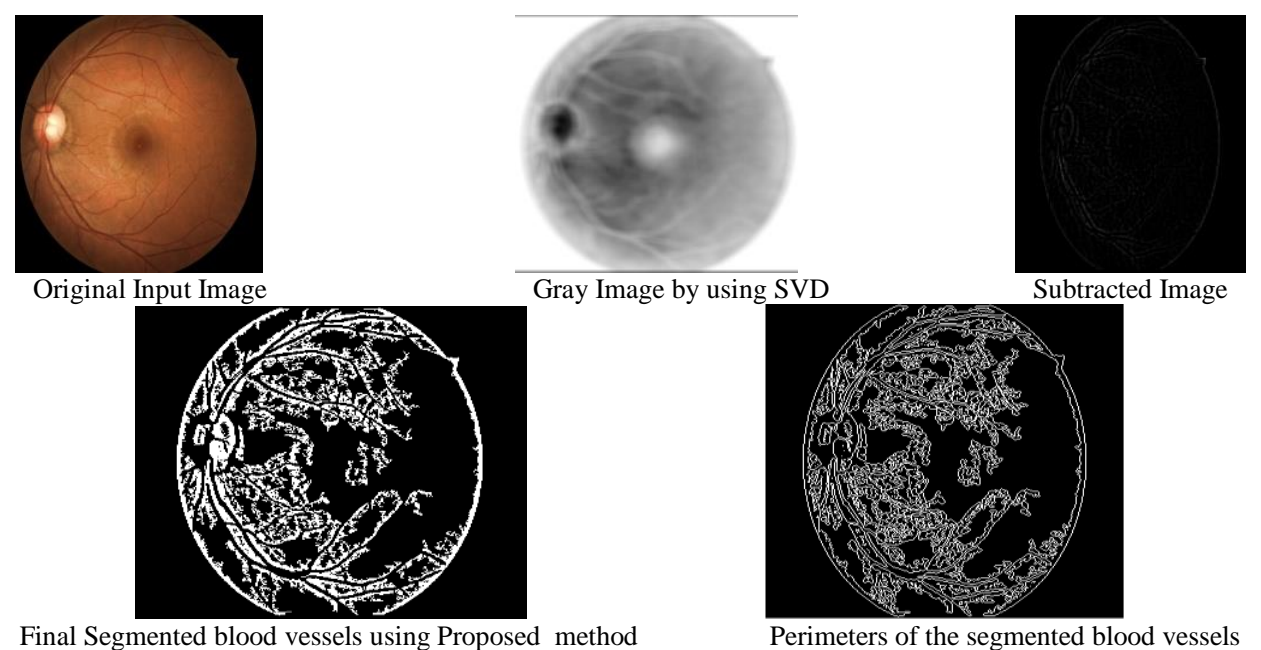

Fig. 2. Segmentation Results of the Proposed Method based on SVD and Morphological Opening on Retinal Images: (a) Depicts the Input Retinal Images with Blood Vessels, (b) Shows the Contrast Enhancement Image using SVD, (c) Depicts the Subtracted Image of Gray an Average Filter, (d) and (e) Shows the Final Blood Vessels Segmentation and its Perimeter of the Resultant Image Respectively. 

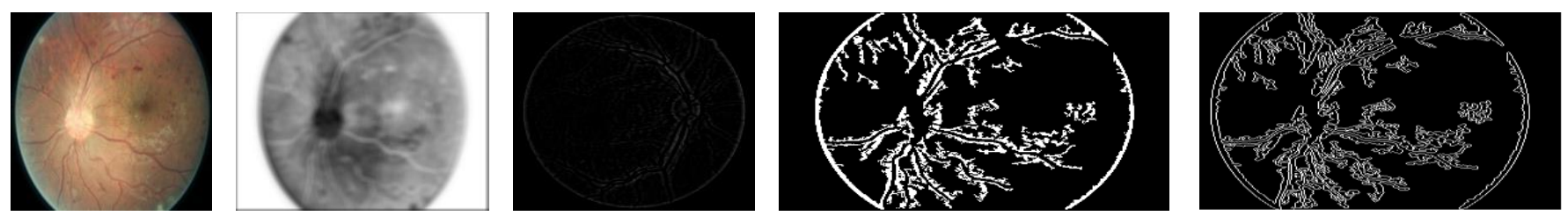

Fig. 3. Segmentation Results of the Proposed Method based on SVD and Morphological Opening on Retinal Images:(a) Depicts the Input Retinal Images with Blood Vessels, (b) Shows the Contrast Enhancement Image using SVD, (c) Depicts the Subtracted Image of Gray and Average Filter, (d) and (e) Shows the Final Blood Vessels Segmentation and its Perimeter of the Resultant Image Respectively.
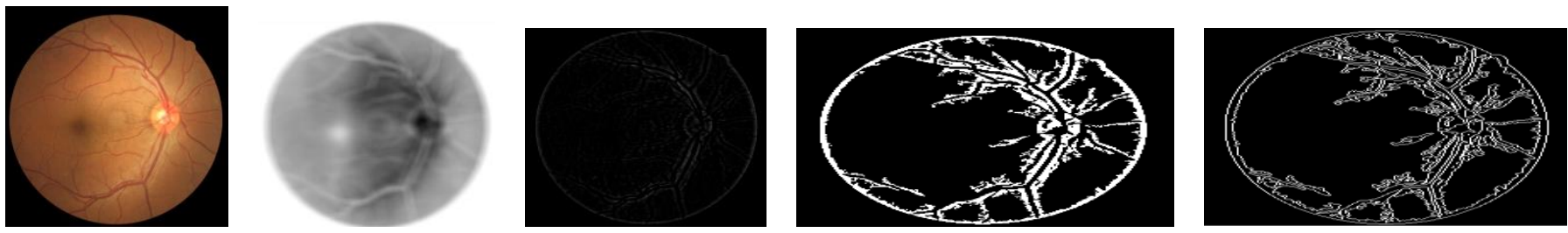

Fig. 4. Segmentation Results of the Proposed Method based on SVD and Morphological Opening on Retinal Images: (a) Depicts the Input Retinal Images with Blood Vessels, (b) Shows the Contrast Enhancement Image using SVD, (c) Depicts the Subtracted Image of Gray and Average Filter, (d) and (e) Shows the Final Blood Vessels Segmentation and its Perimeter of the Resultant Image Respectively.
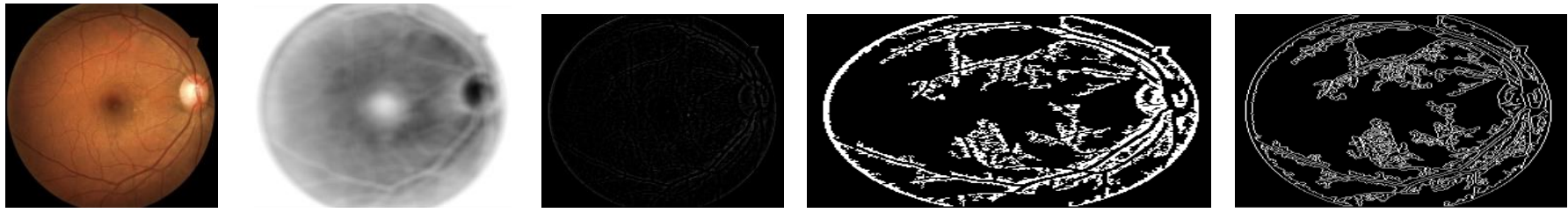

Fig. 5. Segmentation Results of the Proposed Method based on SVD and Morphological Opening on Retinal Images: (a) Depicts the Input Retinal Images with Blood Vessels, (b) Shows the Contrast Enhancement Image using SVD, (c) Depicts the Subtracted Image of Gray an Average Filter, (d) and (e) Shows the Final Blood Vessels Segmentation and its Perimeter of the Resultant Image Respectively.

TABLE. I. Performance Comparison Between Existing Methods and Proposed Method in Terms of Sensitivity, SpeCificity, and Accuracy

\begin{tabular}{|c|c|c|c|c|c|c|c|c|}
\hline No & Author/Authors & Database & Sensitivity & Specificity & TPR & FPR & Accuracy & Technique used \\
\hline \multirow{2}{*}{1} & \multirow{2}{*}{ You et al (2011) [20] } & DRIVE & 0.7410 & 0.9751 & - & - & 0.9434 & \multirow{2}{*}{$\begin{array}{l}\text { Radial projection and semi- } \\
\text { supervised approach. }\end{array}$} \\
\hline & & STARE & 0.7260 & 0.9756 & - & - & 0.9497 & \\
\hline \multirow{2}{*}{2} & \multirow{2}{*}{ Kumar et al (2016) [ 21] } & DRIVE & 0.7006 & 0.9871 & - & - & 0.9626 & \multirow{2}{*}{ Laplacian 2-D Matched Filter } \\
\hline & & STARE & 0.7060 & 0.9693 & - & - & 0.9340 & \\
\hline \multirow{2}{*}{3} & \multirow{2}{*}{ Soares et al (2006) [ 22] } & DRIVE & - & - & 0.7165 & 0.0252 & 0.9480 & \multirow{2}{*}{ MF-FDOG } \\
\hline & & STARE & - & - & 0.7283 & 0.0212 & 0.9440 & \\
\hline \multirow{2}{*}{4} & \multirow{2}{*}{ Mendonca et al (2006) [23 ] } & DRIVE & - & - & 0.6996 & 0.0270 & 0.9466 & \multirow{2}{*}{ MF-FDOG } \\
\hline & & STARE & - & - & 0.7344 & 0.0236 & 0.9452 & \\
\hline \multirow{2}{*}{5} & \multirow{2}{*}{ B. Zhang et al (2010) [24 ] } & DRIVE & 0.7120 & 0.9724 & - & - & 0.9382 & \multirow{2}{*}{$\begin{array}{l}\text { Matched Filter with first order } \\
\text { derivative of Gaussian }\end{array}$} \\
\hline & & STARE & 0.7177 & 0.9753 & - & - & 0.9484 & \\
\hline \multirow{2}{*}{6} & \multirow{2}{*}{$\begin{array}{l}\text { Martinez-Perez et al (2007) } \\
\text { [25] }\end{array}$} & DRIVE & 0.7246 & 0.9655 & - & - & 0.9344 & \multirow{2}{*}{ Multi-scale feature extraction } \\
\hline & & STARE & 0.7506 & 0.9569 & - & - & 0.9410 & \\
\hline \multirow{2}{*}{7} & \multirow{2}{*}{$\begin{array}{l}\text { Mendonca et al (2006) } \\
{[26]}\end{array}$} & DRIVE & 0.7344 & 0.9764 & - & - & 0.9452 & \multirow{2}{*}{$\begin{array}{l}\text { Center lines detection and } \\
\text { morphological reconstruction }\end{array}$} \\
\hline & & STARE & 0.6996 & 0.9730 & - & - & 0.9440 & \\
\hline \multirow{2}{*}{8} & \multirow{2}{*}{$\begin{array}{l}\text { Palomera-Perez et al. (2010) } \\
\text { [27 ] }\end{array}$} & DRIVE & 0.64 & 0.967 & - & - & 0.9250 & \multirow{2}{*}{$\begin{array}{l}\text { Parallel multiscale feature } \\
\text { extraction and region growing }\end{array}$} \\
\hline & & STARE & 0.769 & 0.9449 & - & - & 0.926 & \\
\hline \multirow{4}{*}{9.} & \multirow{4}{*}{ Proposed method } & $\begin{array}{l}\text { STARE } \\
\text { (Fig.2) }\end{array}$ & 0.9123 & 0.9556 & 0.8978 & 0.0092 & 0.9738 & $\begin{array}{l}\text { Singular Valued Decomposition } \\
\text { (SVD) }\end{array}$ \\
\hline & & $\begin{array}{l}\text { STARE } \\
\text { (Fig.3) }\end{array}$ & 0.9443 & 0.9567 & 0.9367 & 0.0008 & 0.9745 & SVD \\
\hline & & $\begin{array}{l}\text { DRIVE } \\
\text { (Fig.4) }\end{array}$ & 0.9468 & 0.9666 & 0.9489 & 0.0007 & 0.9732 & SVD \\
\hline & & $\begin{array}{l}\text { DRIVE } \\
\text { (Fig.5) }\end{array}$ & 0.9567 & 0.9687 & 0.9567 & 0.0005 & 0.9778 & SVD \\
\hline
\end{tabular}


TABLE. II. BLOOD VESSELS SEGMENTATION AREA COVERED By USING PROPOSED METHOD

\begin{tabular}{|l|l|l|l|l|}
\hline $\begin{array}{l}\text { Proposed Method/ } \\
\text { Blood vessels Images }\end{array}$ & $\begin{array}{l}\text { Image 1 } \\
\text { (Fig. 2) }\end{array}$ & $\begin{array}{l}\text { Image 2 } \\
\text { (Fig. 3) }\end{array}$ & $\begin{array}{l}\text { Image 3 } \\
\text { (Fig. 3) }\end{array}$ & $\begin{array}{l}\text { Image 4 } \\
\text { (Fig. 4) }\end{array}$ \\
\hline $\begin{array}{l}\text { Blood vessels } \\
\text { segmentation area } \\
\text { covered }\end{array}$ & $\begin{array}{l}474.4525 \\
\mathrm{~mm}^{2}\end{array}$ & $\begin{array}{l}504.8308 \\
\mathrm{~mm}^{2}\end{array}$ & $\begin{array}{l}519.9923 \\
\mathrm{~mm}^{2}\end{array}$ & $\begin{array}{l}496.3786 \\
\mathrm{~mm}^{2}\end{array}$ \\
\hline
\end{tabular}

\section{CONCLUSION AND FUTURE WORK}

In this paper, a novel algorithm to segment tree-like vascular structure from retinal images is proposed. The procedure has been successful to pull out the required object features of an input image using the SVD's left singular vector matrix for transformation into gray image. This method has been practically proved to be effective in attaining average segmentation accuracy of $97.48 \%$, which is superior compared with existing segmentation algorithms listed in Table I. This new technique got tested upon images contained in DRIVE and STARE databases. In order to attain further accuracy, this technique can be combined with any of the optimization techniques. This combination of the proposed method and an optimization technique will give best results.

\section{ACKNOWLEDGMENT}

I thank my guide, faculty, Head of the Department, Principal of GITAM Institute of Technology (GIT) for their kind support and encouragement to carry out this work.

\section{REFERENCES}

[1] G. J.J. Kanski, Clinical Ophthalmology, 6th ed., ElsevierHealth Sciences, London, UK, 2007.

[2] K. Verma, P. Deep, A.G. Ramakrishnan, "Detection and classification of diabetic retinopathy using retinal images flight', Annual IEEE India Conference (INDICON), vol. 4, pp. 1-6, 2011.

[3] M.M. Fraz, P. Remagnino, A. Hoppe, B. Uyyanonvara, A.R. Rudnicka, C.G. Owen, S.A. Barman, "Blood vessel segmentation methodologies in retinal images a survey", Comput. Methods Programs Biomed, vol. 1, pp. 407-433, 2012.

[4] T. Walter, P. Massin, A. Erginay, R. Ordonez, C. Jeulin, J.C. Klein, Automatic detection of microaneurysms in color fundus images, Med. Image Anal. 11 (2007) 555-566.

[5] D. Kahaner, C. Moler and S. Nash, Numerical Methods and Software, Prentice-Hall, Inc, 1989.

[6] https://en.wikipedia.org/wiki/RGB_color_model.

[7] Tarun Kumar, Karun Verma "A Theory Based on Conversion of RGB image to Gray image" International Journal of Computer Applications (0975- 8887) Volume 7- No.2, September 2010.J. Clerk Maxwell, A Treatise on Electricity and Magnetism, 3rd ed., vol. 2. Oxford: Clarendon, 1892, pp.68-73.

[8] https://www.xrite.com/blog/lab-color-space.

[9] Saravanan, C. (2010). Color Image to Grayscale Image Conversion. 2010 Second International Conference on Computer Engineering and Applications.doi:10.1109/iccea.2010.192.
[10] T. Walter, P. Massin, A. Erginay, R. Ordonez, C. Jeulin, J.C. Klein, Automatic detection of microaneurysms in color fundus images, Med. Image Anal. 11 (2007) 555-566.

[11] C. Tyler, "A Novel Retinal Blood Vessel Segmentation Algorithm for Fundus Images," MATLAB Cent. File Exch., 2016.

[12] Du, H., He, S., Sheng, B., Ma, L., \& Lau, R. W. H. (2015). SaliencyGuided Color-to-Gray Conversion Using Region-Based Optimization. IEEE Transactions on Image Processing, 24(1), 434443.doi:10.1109/tip.2014.2380172.

[13] DibyaJyoti Bora, "Importance of Image Enhancement Techniques in Color Image Segmentation: A comprehensive and Comparative Study", Indian J.Sci.Res. 15, vol. 4, pp. 115-131, 2017.

[14] Mithilesh Kumar, AshimaRana, "Image Enhancement using Contrast Limited Adaptive Histogram Equalization and Wiener filter", International Journal Of Engineering And Computer Science, vol. 5, pp. 16977-16979, 2016

[15] K. Bhargavi, S. Jyothi, "A Survey on Threshold basedSegmentation Technique in Image Processing", International Journal Of Innovative Research and Development, ( Vol 3 Issue 12, November, 2014.

[16] https://www.harrisgeospatial.com/docs/ISODATAClassification.html.

[17] https://in.mathworks.com/help/images/morphological-dilation-anderosion.html.

[18] Niemeijer M, Staal JJ, GinnekenBv, Loog M, Abramoff MD.DRIVE: digital retinal images for vessel extraction; 2004. WebLink:http://www.isi.uu.n1/Research/Databases/DRIVE/.

[19] The STARE project, [online] Available: http: //www.ces.clemson.edu/ ahoover/stare.

[20] You, X., Peng, Q., Yuan, Y., Cheung, Y., \& Lei, J. (2011). Segmentation of retinal blood vessels using the radial projection and semi-supervised approach. Pattern Recognition, 44(10-11), 23142324.doi:10.1016/j.patcog.2011.01.007.

[21] Kumar, D., Pramanik, A., Kar, S. S., \&Maity, S. P. (2016). Retinal blood vessel segmentation using matched filter and Laplacian of Gaussian. 2016 International Conference on Signal Processing and Communications (SPCOM). doi:10.1109/spcom.2016.7746666.

[22] J.V.B. Soares, J.J.G. Leandro, R.M. Cesar-Jr., H.F. Jelinek, and M.J. Cree, "Retinal vessel segmentation using the 2-d gabor wavelet and supervised classification," IEEE Trans. on Medical Imaging, vol. 25, pp. 1214-1222, 2006.

[23] Mendonca, A. M., \&Campilho, A. (2006). Segmentation of retinal blood vessels by combining the detection of centerlines and morphological reconstruction. IEEE Transactions on Medical Imaging, 25(9), 12001213. doi:10.1109/tmi.2006.879955.

[24] B. Zhang, L. Zhang, L. Zhang, F. Karray, Retinal vessel extraction by matched filter with first-order derivative of Gaussian, Computers in Biology and Medicine 40 (2010)438-445.

[25] M.E. Martinez-Perez, A.D. Hughes, S.A. Thom, A.A. Bharath, K.H. Parker, Segmentation of blood vessels from red-free and fluorescein retinal images, Medical Image Analysis 11 (2007) 47-61.

[26] Safsfd A.M. Mendonca, A. Campilho, Segmentation of retinalblood vessels by combining the detection of centerlines and morphological reconstruction, IEEE Transactions on Medical Imaging 25 (2006) 12001213.

[27] Palomera-Perez, M. A., Martinez-Perez, M. E., Benitez-Perez, H., \& Ortega-Arjona, J. L. (2010). Parallel Multiscale Feature Extraction and Region Growing: Application in Retinal Blood Vessel Detection. IEEE Transactions on Information Technology in Biomedicine, 14(2), 500506.doi:10.1109/titb.2009.2036604. 